\title{
Towards a New Approach to Rationalize the Operators' Choice in Telecommunication Networks
}

\author{
Driss Ait Omar ${ }^{1}$, Mohamed Baslam ${ }^{2}$, Mourad Nachaoui ${ }^{3}$ and Mohamed Fakir ${ }^{4}$ \\ ${ }^{1,2,4}$ Information Processing and Decision Support Laboratory, Faculty of Sciences \\ and Technics, University of Sultan Moulay Slimane, Beni Mellal, Morocco \\ ${ }^{3}$ Mathematics and Applications Laboratory, Faculty of Sciences \\ and Technics, University of Sultan Moulay Slimane, Beni Mellal, Morocco \\ 1aitomard@gmail.com, ${ }^{2}$ baslam.med@gmail.com, ${ }^{3}$ nachaoui@gmail.com, \\ ${ }^{4}$ fakfad@yahoo.fr
}

\begin{abstract}
Optimization is an essential tool in the field of decision support. In this paper, we interested to study an inverse problem applied in the telecommunication networks. Indeed, in the telecommunication networks, service providers have subscription offers to customers. Since competition is strong in this sector, most of these advertising offerings, totally or partially ambiguous, are prepared to attract the attention of consumers. For this reason, customers face problems in making decisions about the choice of the operators that gives them a better report price/QoS. Mathematical modeling of this decision support problem led to the resolution of an inverse problem. More precisely, the inverse problem is to find the function of the QoS real knowing the QoS theoretical or advertising. This model will help customers who seek to know the degree of sincerity of their operators, as well as it is an opportunity for operators who want to maintain their resources so that they gain the trust of customers. We have used the Genetic Algorithm to resolve the optimization problem proposed in our work. We were able to learn that when the SP bandwidth increase their weight in the system increase, because they able to offers a better quality of services. However, when their demand increase without changing the bandwidth, the quality of services decreases with their weight.
\end{abstract}

Keywords: Telecommunication Networks, QoS, Pricing, Inverse problem, Optimization, Genetic Algorithm

\section{Introduction}

In the recent years, the privatization and liberation of services in telecommunication networks sectors lead to a diversification of services providers. This diversity leads to strong competition between them, each of which tries to attract and / or retain customers. Since do not give true information about their systems (client confusion), customers do not have complete information to make a good decision. This confusion present an obstacle to customers to have all the information on the operator's offer. For it, consumer confusion has been studied as an important in many markets due to dynamics, such as over choice, excessive marketing communications, and similar tariffs and promotions. Hence, in the presence of confusion, the customer's choice is often uncertain (bounded rationality).

Telecommunication networks also have been considered in the economic literature as one of the sectors that causes confusion among consumers [13]. Complex fee structures introduced by service providers, number portability, loyalty

Received (March 10, 2018), Review Result (May 10, 2018), Accepted (May 15, 2018) 
programs and complicated service variations make it difficult for consumers to understand the type of transaction and get the best report price /QoS.

The problems related to the choice of an operator is on several parameters including real quality of service, theoretica service quality, bandwidth, price... Operators decide a price and QoS for services offered to its customers. QoS proposed remains a parameter that depends on other variables namely bandwidth, the share of this market operator. In these circumstances, an operator can never guarantee the quality of service it promises to customers. We call it then theoretical QoS, while the QoS perceived by customers is the real QoS. In the telecommunications networks, the credibility of each operator is measured by the difference between its theoretical QoS and the real QoS. A customer is interested in the recognition of with good credibility (which has a real QoS close to the theoretical QoS). In addition, as there is no real QoS to operators cannot solve this problem in a direct way. Hence, this kind of problem can be modeled within the meaning of the inverse problem. The inverse problem is generally ill-posed problem, on the contrary live with the problem said that the solution exists, is unique and depends on data. For example, if it is to reconstruct the past state of a system knowing its current state, we are dealing with an inverse problem; but the fact of predicting the future state given the current state is a direct problem. Similarly, in the case of a determination of parameters of a system knowing a part of the stage (a part of the set of parameters); we speak of parameter identification problems.

In related work, many studies have contributed to the study of the problem of consumer confusion and their bounded rationality. However, to my knowledge there is no work that is interested in finding the form of real QoS to know the confidence level of service providers in telecommunication networks. The authors of the paper [13] they proposed a model that is based on the economic weighing of and the Luce probabilistic choice model. They concluded that the client is confused or not by the SPs, if its degree of irrationality increases, the earns more; this model of Luce's choice is often more complicated than the discrete choice model implied by the authors of the paper [14]-[16]. In addition, the authors of the book [15] debate the confusion concept, display that irrational decision-making can be explained if one takes into account that human capacities to process information are limited. Thus, they used and improved heuristic techniques to predict the quality of the decision by formulating and solving optimization problems. The use of heuristic methods for the optimization of the proposed models is due to their efficiency in the search for the global optimum.

The instead of this paper is organized as follows: in Section 2, we present modeling problematic using the inverse problem theory. We present in Section 3 the genetic algorithm as optimization method used to solve the models proposed in our work. In Section 4, we present the different numerical results obtained. We conclude this study with perspectives in Section 5.

\section{Formulation and Modeling of the Problem}

In this section, we model the profit of a customer if he chooses to subscribe in the $S P_{i}$ and we use the Luce model to mathematise the discrete choice of clients by exploiting the softmax function or the normalized exponential function [11], as in the article [10]. 


\subsection{Model of Utility Customers}

The benefit of a consumer is often calculated based on the strategies of its operator, which are the QoS and the price it offers. The profit $u_{i}$ of a consumer registers with the operator's services $S P_{i}$ is as follows:

$$
u_{i}\left(q_{i}, p_{i}\right)=\alpha \cdot q_{i}-p_{i}
$$

with $\alpha q_{i}$ is a client's revenue if he chooses the $\boldsymbol{S P}_{\boldsymbol{i}}$ and $\boldsymbol{\alpha}>0$.

\subsection{The Luce Model}

The Luce model is a first probabilistic choice model that incorporates boundedly rational choice of customers[1]-[2]-[13]. With this model, customers can choose the operator that will maximize profits by choosing one that has the maximum probability; but forcing this choice is inadequate in this area given the existence of hidden information that has not been represented in this model. The following equation represents the probability that the customer chooses $S P_{i}$ :

$$
\rho_{i}(p, q)=\frac{\mathrm{e}^{\frac{u_{i}}{\lambda}}}{\sum_{j=1}^{N} \mathbf{e}^{\frac{u_{j}}{\lambda}}}
$$

with $\lambda \in[0,1]$ is the degree of customer irrationality, $N$ is the number of SPS and $\boldsymbol{p}$ and $\boldsymbol{q}$ are respectively the vector of price and QoS.

When $\lambda$ tends to 0 , means that customer behavior is rational, i.e., they have all the information and rules that allow them to maximize their profit, while customers are irrational when $\lambda$ approaches 1 .

\subsection{Demand Model $D_{i}$}

We consider a market size $\mathrm{n}$ (the total number of customers), the function of the application to the operator's services $i, \boldsymbol{D}_{\boldsymbol{i}}$ is the probability that a customer selects the operator multiplied by the size, $\mathrm{n}$, of the market. It is expressed by:

$$
D_{i}(p, q)=n \cdot \rho_{i}(p, q)
$$

\subsection{Theoretical Quality of Service}

We consider Delay $y_{i}^{u}$ the time required for data transmission to a $S P_{i}$ user $u$. This time is expressed in telecommunications [3] as a function of bandwidth available at $S P_{i}$ and the demand $D_{i}$ :

$$
\operatorname{Delay}_{i}^{u}=\frac{1}{\Phi_{i}-D_{i}}
$$

That means more demand is greater than the time increases, and vice versa, over the bandwidth increases the time becomes less important. This proportionality is logical since:

- More as demand increases, the number of customers connected to the $S P_{i}$ becomes large and thus the time becomes more important. 
- More than the bandwidth increases, the $S P$ largely has capacity to cover all customers and therefore the time became smaller.

In the model of L. Kleinrock [8] with queues, Quality of service QoS is the inverse of the total response time when the user wants to access the service. Let $c_{i}$ the deadlines for transmission of data between the service provider and the provider services l' $S P_{i}$, the total time of the answer is accumulates between $c_{i}$ and Delay $y_{i}^{u}$. Thus the quality of service is expressed by the following equation (as [4]):

$$
q_{i}=\frac{1}{\text { Delay }_{i}^{u}+c_{i}}
$$

From the two equations (4) et (5), we show the existence of the relationship between the quality of service, demand $\boldsymbol{D}_{\boldsymbol{i}}(\boldsymbol{p}, \boldsymbol{q})=\boldsymbol{n} \boldsymbol{\rho}_{\boldsymbol{i}}(\boldsymbol{p}, \boldsymbol{q})$ and bandwidth $\Phi_{i}$ by the following equation [3]:

$$
\boldsymbol{q}_{i}=\frac{\Phi_{i}-\boldsymbol{D}_{i}}{1+\boldsymbol{c}_{i}\left(\Phi_{i}-\boldsymbol{D}_{i}\right)}
$$

or by the following equation:

$$
\Phi_{i}=D_{i}+\frac{q_{i}}{1-q_{i} c_{i}}
$$

From the equations (6), we can deduce that when demand of $S P_{i}$ approach covering all the bandwidth; QoS becomes less.

\subsection{Problem Formulation}

From equation (1), we find that the theoretical benefit of a user is:

$$
u_{i}\left(q_{i}, p_{i}\right)=\alpha \cdot q_{i}-p_{i}
$$

then the real benifit is:

$$
u_{i}^{R}\left(q_{i}^{R}, p_{i}^{R}\right)=\alpha \cdot q_{i}^{R}-p_{i}^{R},
$$

with $\boldsymbol{q}_{i}^{\boldsymbol{R}}$ is a real QoS and $\boldsymbol{p}_{i}^{\boldsymbol{R}}$ is a real price.

Assumption 1 In telecommunications, there is no general difference between promotional price (theoretical) and the real price that the user paid when the invoice settlement. However, in our study we assume that $\boldsymbol{p}_{i}=\boldsymbol{p}_{i}^{\boldsymbol{R}}$.

Taking into consideration this assumption 1 , the difference between the real and theoretical benefit becomes:

$$
u_{i}-u_{i}^{R}=\alpha . q_{i}-p_{i}-\left(\alpha . q_{i}^{R}-p_{i}^{R}\right)=\alpha\left(q_{i}-q_{i}^{R}\right)
$$

\subsubsection{Resource Management Model}

Used to help operators in the telecommunications field to maintain their resources so that the difference between their offers and advertising that benefit the customer actually is optimal. It is a tool to customers who want to register with the operator that meets their needs.

$$
\min \boldsymbol{F}_{i}(\boldsymbol{p}, \boldsymbol{q})=\frac{1}{2} \cdot \boldsymbol{\alpha} \cdot \boldsymbol{\rho}_{i} \cdot\left[\left(\boldsymbol{q}_{i}-\boldsymbol{q}_{i}^{R}\right)^{2}\right]
$$


under the constraints:

$$
\left\{\begin{array}{l}
\boldsymbol{p}_{i}<\boldsymbol{P}_{\text {max }} \\
\boldsymbol{q}_{\text {min }} \triangle \boldsymbol{q}_{i}^{R} \square \boldsymbol{q}_{i}
\end{array}\right.
$$

The first constraint is related to customer purchasing power. the second constraint is a formulation in terms of customer needs in real QoS. it must meet a minimum threshold and it should not exceed the theoretical QoS.

\subsubsection{Discrete Choice Model Customers}

Lets customers know the weight (sincerity) of all operators in the telecommunications market. This problem is formulated as a this multi-objective model:

$$
\min G(p, q)=\left(\begin{array}{c}
F_{1}(p, q) \\
F_{2}(p, q) \\
\vdots \\
F_{N}(p, q)
\end{array}\right)
$$

under the constraints:

$$
\left\{\begin{array}{l}
\forall i \in\{1, \ldots, N\} \boldsymbol{p}_{i}<\boldsymbol{P}_{\text {max }} \\
\forall \boldsymbol{i} \in\{1, \ldots, N\} \boldsymbol{q}_{\text {min }} \square \boldsymbol{q}_{i}^{R} \square \boldsymbol{q}_{i}
\end{array}\right.
$$

To solve the multi-objective problem (MOP), we must transform it into a singleobjective problem weighted. For this, we applied the aggregation method and the result of the transformation is:

$$
\min G(p, q)=\sum_{i=1}^{N} \gamma_{i} \cdot F_{i}(p, q)
$$

under the constraints:

$$
\left\{\begin{array}{l}
\forall i \in\{1, \ldots, N\} 0 \square \gamma_{i}<\llbracket t \sum_{i=1}^{N} \gamma_{i}=1 \\
\forall i \in\{1, \ldots, N\} p_{i}<\boldsymbol{P}_{\text {max }} \\
\forall i \in\{1, \ldots, N\} \boldsymbol{q}_{\min } \Delta \boldsymbol{q}_{i}^{\boldsymbol{R}}<\boldsymbol{q}_{i}
\end{array}\right.
$$

and

$$
\boldsymbol{F}_{i}(\boldsymbol{p}, \boldsymbol{q})=\frac{1}{2} \cdot \boldsymbol{\alpha} \cdot \rho_{i} \cdot\left[\left(\boldsymbol{q}_{i}-\boldsymbol{q}_{i}^{\boldsymbol{R}}\right)^{2}\right], \boldsymbol{i}=1,2, \ldots, \boldsymbol{N}
$$

With $\gamma$ is considered vector weight of the operators in the telecommunications market.

\section{Genetic Algorithm}

Genetic Algorithms (GAs) developed by Holland [9] and his student Goldberg [6], are based on the mechanics of natural evolution and natural genetics[5]-[7]. GAs differ from usual inversion algorithms because they do not require a starting value. The GAs use a survival-of-the-fittest scheme with a random organized search to find the best solution to a problem. Solve an optimization problem is find the optimum of a function from a finite number of choices, often very large. The practical applications are numerous, whether in 
the field of industrial production, transport or economics - wherever there is need to minimize or maximize digital functions in systems simultaneously operate a large number of parameters. Algorithm (1) represents the genetic algorithm used to optimize the models proposed in this work.

Algorithm 1: Genetic Algorithm

1. Initialize the initial population $\mathbf{P}$.

2. Evaluate $P(t)$.

3. While No convergence do

4. a. $P(t+1)=$ Selection of Parents in $P(t)$.

b. $P(t+1)=$ Apply Crossing Operator on $P(t+1)$

c. $P(t+1)=$ Apply on Mutation Operator $P(t+1)$

d. $P(t)=$ Replace elders of $P(t)$ Descendants of their $P(t+1)$

e. Evaluate $P(t)$

5. End while

\section{Numerical Results}

In this section, we present the numerical results obtained by assuming that we have $S P \mathrm{~s}$ in this telecommunications market. We use the genetic algorithm with the parameters that will allow us to obtain the optimal solution for our proposed models.

\subsection{The Real Quality of the Function Study}

\subsubsection{Study of a Limited Case}

In the telecommunications market, the real quality of service is a function that depends on the bandwidth $\Phi_{i}$ and the demand $\boldsymbol{D}_{\boldsymbol{i}}$ of $\boldsymbol{S} \boldsymbol{P}_{\boldsymbol{i}}$. In reality, we know that when the bandwidth increases, the real quality of service (QoS) increases and vice versa; also when demand increases, real service quality decreases and increases when demand decreases. In this context, we observed that the real quality can be expressed as a polynomial of degree 2, the variable $x_{i}$ is the ratio between $\Phi_{i}$ and $\boldsymbol{D}_{i}$, as following:

$$
\boldsymbol{q}_{i}^{R}\left(\Phi_{i}, \boldsymbol{D}_{i}\right)=\alpha_{i}^{1} *\left(\frac{\Phi_{i}}{\boldsymbol{D}_{i}}\right)^{2}+\boldsymbol{\alpha}_{i}^{2} *\left(\frac{\Phi_{i}}{\boldsymbol{D}_{i}}\right)+\boldsymbol{\alpha}_{i}^{3}
$$

We used the genetic algorithm (with the table settings 1) to find these coefficients for different values of bandwidth $\Phi_{i}$ and of demand $\boldsymbol{D}_{\boldsymbol{i}}$. The Figures 1 et 2 show the influence of respectively $\Phi_{i}$ and $\boldsymbol{D}_{i}$ on QoS (theorical and real).

Table 1. Genetic Algorithm Parameters to the Figures of the Results 1 et 2

\begin{tabular}{|l|l|}
\hline Population size $\mathbf{N}$ & 16 \\
\hline Type selection & at roulette \\
\hline Type of crossover & Single-points \\
\hline Probability of crossover $P_{c}$ & 0.7 \\
\hline Type of mutation & uniform \\
\hline Probability of mutation & 0.05 \\
\hline Maximum number of generations & 100 \\
\hline
\end{tabular}




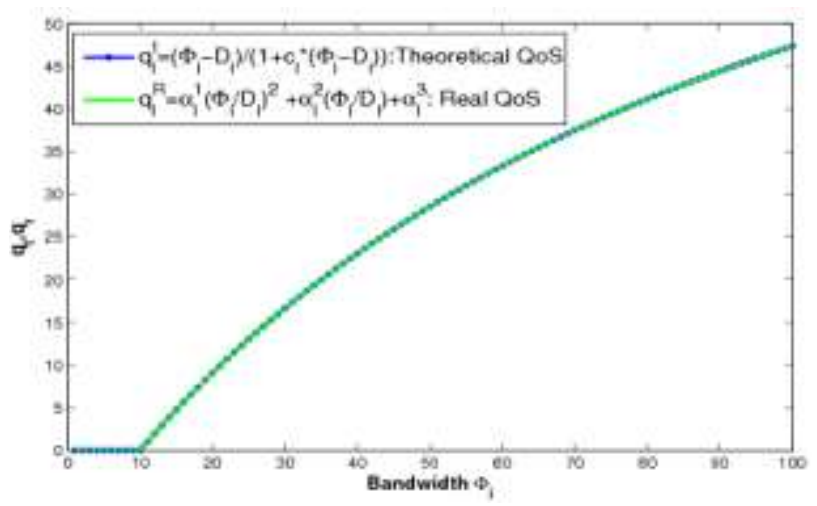

Figure 1. Variation of $q_{i}^{t}$ and $q_{i}^{R}$ in Terms of $\Phi_{i}$

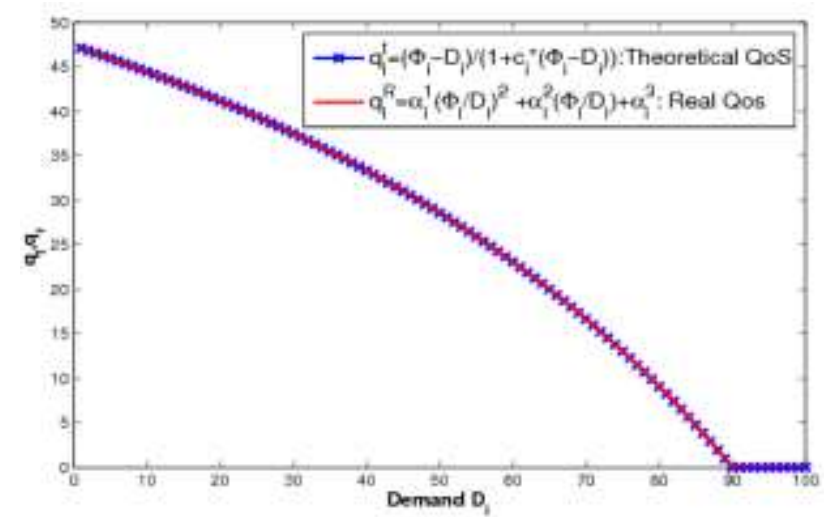

Figure 2. Variation of $q_{i}^{t}$ and $q_{i}^{R}$ in Terms of $D_{i}$

From Figures (1 et 2) we note that with the change of bandwidth $\Phi_{i}$ and the demand $\boldsymbol{D}_{\boldsymbol{i}}$, the genetic algorithm was able to find the good coefficients of the polynomial to minimize the gap between what is theoretical and what is real. In the next part, we will not restrict ourselves to the case presented above, but we are expanding the study of the variation of the actual quality using the technique of discretization dominates definition of theoretical quality seeking at each point the value of the actual quality by solving the model for managing resources.

\subsubsection{Study of a General Case by Discretization}

The quality theoretical of a $S P_{i}$ may vary within a range delimited by a minimum and maximum value: $\boldsymbol{q}_{i}^{t} \in\left[\boldsymbol{q}_{\min }, \boldsymbol{q}_{\max }\right]$. To make a digital resolution, we will discretize the interval 3, that is to say, turn it into an approximate problem (discrete) to find the values of the actual quality $\boldsymbol{q}_{i}^{R}$ at each point of the discrete domain.

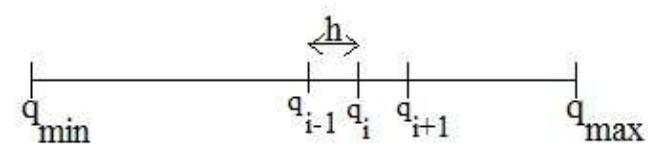

Figure 3. Discretization of an Interval

or $\boldsymbol{h}$ is a positive regular pitch. 


\section{A) Study The Impact of Bandwidth on $q_{t}$ and $q_{r}$}

We launched the genetic algorithm; Matlab programmed with the parameters listed in Table 2; on the model of resource management with variation of bandwidth $\Phi_{i}$, and we obtain the results shown in Figures 4, 5 and Table 3.

\section{Table 2. Genetic Algorithm Parameters to the Figures of the Results 4 and 5}

\begin{tabular}{|l|l|}
\hline Population size $\mathbf{N}$ & 20 \\
\hline Type selection & at roulette \\
\hline Type of crossover & Multi-point \\
\hline Probability of crossover $\boldsymbol{P}_{\boldsymbol{c}}$ & 0.65 \\
\hline Type of mutation & non uniform \\
\hline Probability of mutation & 0.05 \\
\hline Maximal Number of generation & 300 \\
\hline
\end{tabular}

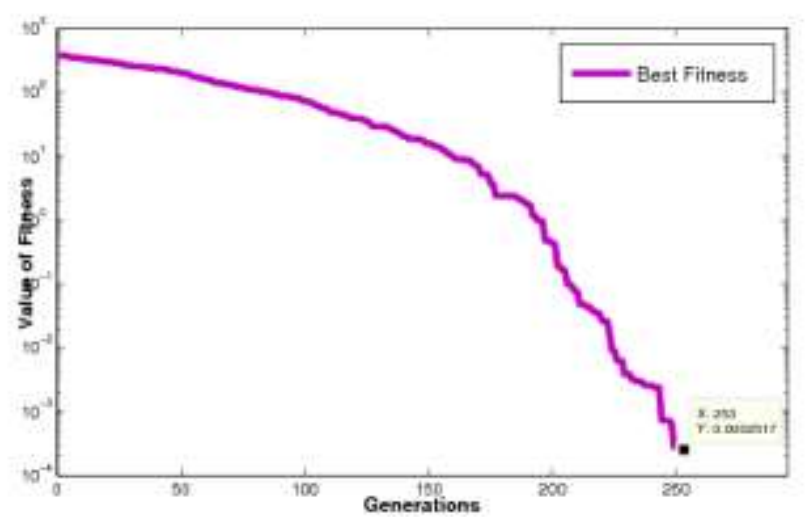

Figure 4. Decrease in the Objective Function According Generations

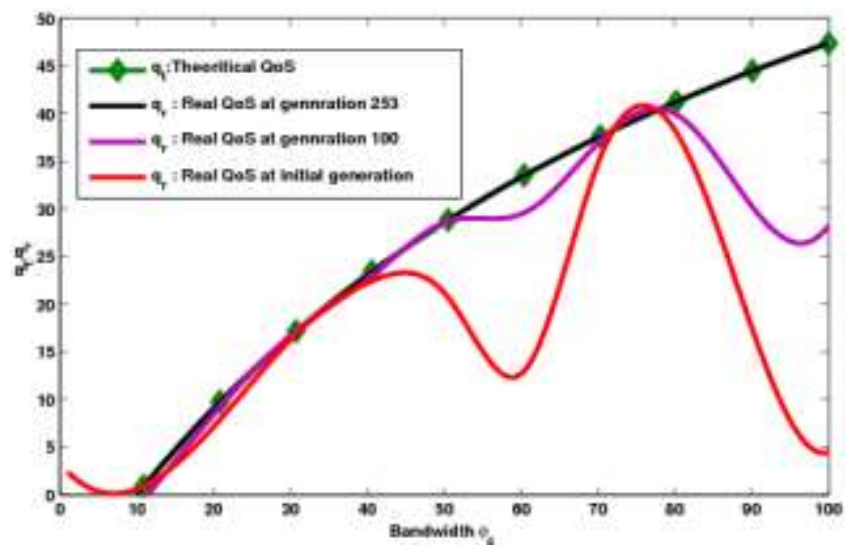

Figure 5. Changes in the Real Quality of Service Over the Generations

Figure 4 shows the decrease of the function Fitness relative to iterations (generations) the genetic algorithm. The objective function value begins $10^{3}$, in the first generation, to reach the value $10^{-4}$, in the $253^{\text {ème }}$ generation. This result shows that the decrease is Igue (remarkable). From Figure 5, we note that to achieve the same minimum value, the algorithm needs to go to the 253 generation. 
Table 3. Convergence Results of the Genetic Algorithm (variation of $\Phi_{i}$ )

\begin{tabular}{|c|c|}
\hline Number of generation & 253 \\
\hline minimum cost & $2.517 * 10^{-4}$ \\
\hline step of discretization $\boldsymbol{h}$ & $\frac{1}{10}$ \\
\hline
\end{tabular}

B) Impact on Demand Study $q_{t}$ and $q_{r}$

We launched the genetic algorithm; with the parameters listed in Table 4;on the model of resource management with variation of the demand $D_{i}$, and we obtain the results shown in the following Figures 6, 7 and Table 5.

Table 4. Genetic Algorithm Parameters to the Figures of the Results 6 and 7

\begin{tabular}{|l|l|}
\hline Population size $\mathbf{N}$ & 10 \\
\hline Type of selection & at roulette \\
\hline Type of crossover & Multi-point \\
\hline Probability of crossover $\boldsymbol{P}_{\boldsymbol{c}}$ & 0.60 \\
\hline Type of mutation & not uniform \\
\hline Probability of mutation & 0.05 \\
\hline Maximum number of generation & 200 \\
\hline
\end{tabular}

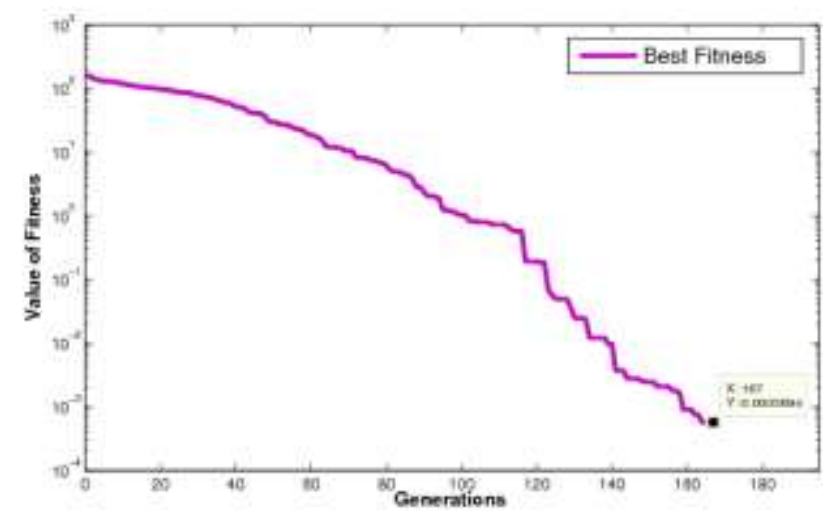

Figure 6. Decrease in the Objective Function According Generations

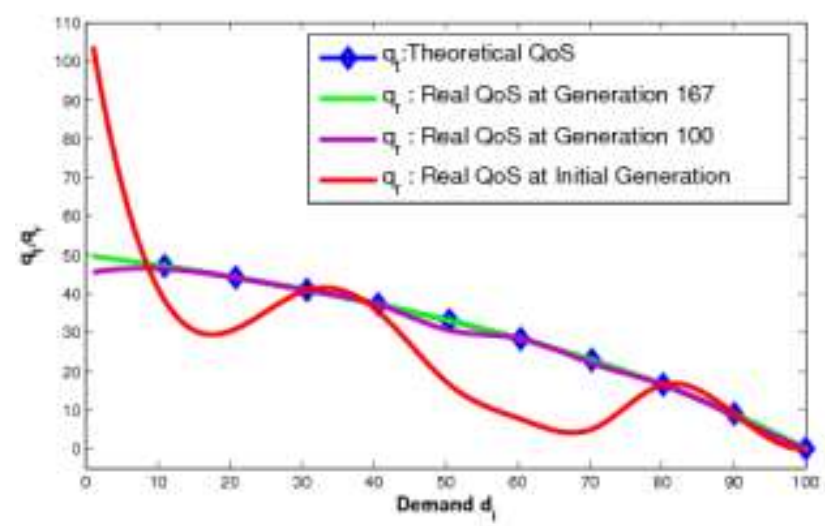

Figure 7. Changes in the Real Quality of Service Over the Generations 
Figure 6 shows the decrease of the function Fitness relative to iterations (generations) the genetic algorithm. the objective function value begins with $10^{2}$ in the first generation, reaching the values $5,694^{*} 10^{-4}$, in the $167^{\text {th }}$ generation. This result shows that the decrease is Igue (remarkable). From the Figure 7, We note that to achieve the same minimum value, the algorithm needs to go to the 167 generation.

\section{Table 5. Convergence Results of the Genetic Algorithm (variation of $\boldsymbol{D}_{i}$ )

\begin{tabular}{|c|c|}
\hline Number of generation & 167 \\
\hline Minimum cost & $5.694 * 10^{-4}$ \\
\hline Step of discretization $\boldsymbol{h}$ & $\frac{1}{20}$ \\
\hline
\end{tabular}

\subsection{Model Resolution of the Weight Calculation}

In this part, we consider a telecommunication network system that we have two services providers. We will use the model of discrete choice of customers to find their weight in this market ranging $\Phi_{i}$ and $\boldsymbol{D}_{i}$ an operator. The calculation of these weights is a kind of decision support for customers seeking to register with the services of the most sincere operator (who has more confidence in the sense of the difference between $\boldsymbol{q}^{t}$ and $\boldsymbol{q}^{\boldsymbol{R}}$.

\subsubsection{Impact of Bandwidth $\Phi$ on the Weight of Operators}

We vary the bandwidth of the operator 1 and observe the influence on weight and that of the adversary. Figure 8 show that the weight of the operator is an increasing function compared to bandwidth $\phi_{1}$, then the weight of its adversary is a decreasing function with respect to $\phi_{1}$. This result is real, since the increase in bandwidth $\phi_{1}$ causes the improvement of the real $\mathrm{QoS} q_{1}^{R}$ and therefore the operator 1 must have a good reputation and a good weight for his adversary.

\subsubsection{Impact of Demand $D$ on the Weight of Operators}

We will vary the request of the operator 1 and observe the influence on weight and that of the adversary. Figure 9 shows that the wight of operator 1 is decreasing function compared to demand $\boldsymbol{D}_{1}$, then the wight of its adversary is a increasing function compared to $\boldsymbol{D}_{1}$. This result is real, because the increase in demand $\boldsymbol{D}_{1}$ causes degradation of the real $\operatorname{QoS} \boldsymbol{q}_{1}^{\boldsymbol{R}}$ and therefore the operator 1 must not have a good reputation and a good weight for his adversary. 


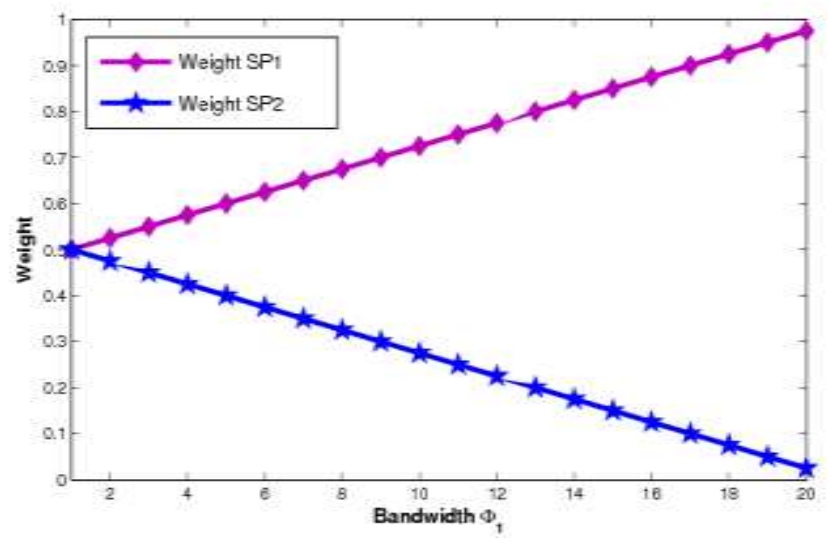

Figure 8. Change in Weight of $S P S$ Increasing $\Phi_{1}$

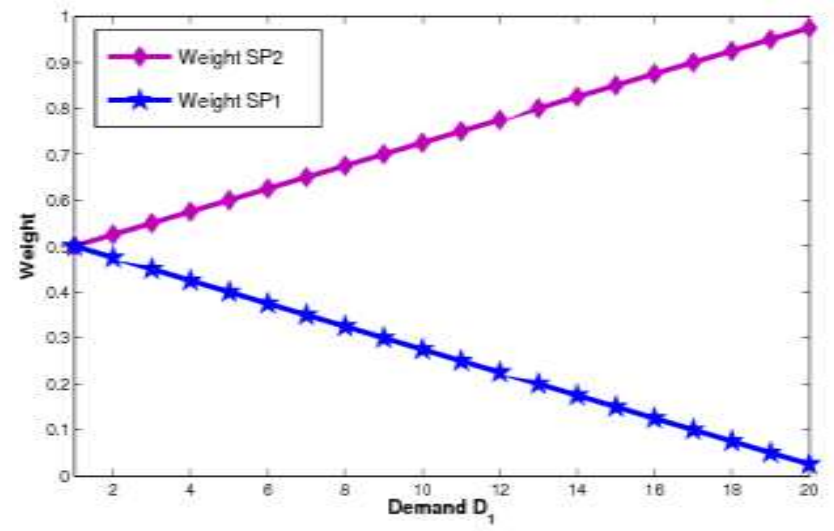

Figure 9. Change in Weight $S P S$ Increasing $D_{1}$

\section{Conclusion}

In this work, we have studied the problem of real quality, which is the key to rational customer decision-making in telecommunications networks. We were able to propose an inverse problem reformulated in the form of optimization models. These models will allow customers to know the service provider that best meets their requirements by knowing the demand and the bandwidth they use. Also, it is a modeling that allows service providers to adapt their bandwidth to user demand so that the difference between what is theoretical and what is perceived is optimal. However, we used the genetic algorithm for the numerical solution of optimization models. This choice is due to the efficiency it shows in the field of global optimization.

In future work, we aim to merge our model with the study of cache in information centric networks [12] to understand the impact of caching on the quality of service perceived by customers.

\section{References}

[1] H.-S. Kim and C.-H. Yoon, "Determinants of subscriber churn and customer loyalty in the Korean mobile telephony market", Telecommunications Policy, vol. 28, (2004), pp. 751-765.

[2] J. Qi, Y. Zhang, Y. Zhang and S. Shi, "Treelogit model for customer churn prediction", in APSCC 06: Proceedings of the 2006 IEEE AsiaPacific Conference on Services Computing. Washington, DC, USA: IEEE Computer Society, (2006), pp. 7075.

[3] M. Baslam, R. El-Azouzi, E. Sabir and L. Echabbi, "Market Share Game with adversarial Access providers : A Neutral and a Non-neutral Network Analysis", Roberto Cominetti and Sylvain Sorin and 
Bruno Tuffin. NetGCOOP 2011: International conference on NETwork Games, COntrol and OPtimization, Paris, France, IEEE, (2011) October, pp. 5.

[4] M. Baslam, L. Echabbi, R. E. Azouzi and E. Sabir, "Joint price and qos market share game with adversarial service providers and migrating customers", Proc. of GameNets, Shanghai, China, (2011) April.

[5] Z. Michalewicz, "Genetic algorithms + data structures = evolution programs", SpringerVerlag, Berlin, second edition, (1994).

[6] D. E. Goldberg and J. H. Holland, "Genetic algorithms and machine learning", Machine learning, vol. 3, no. 2, (1988), pp. 95-99.

[7] K. Deep and M. Thakur, "A new mutation operator for real coded genetic algorithms", Appl. Math. Comput., vol. 193, no. 1, pp. 211-230, (2007).

[8] L. Kleinrock, "Queuing systems”, Wiley Interscience, (1975).

[9] J. H. Holland, "Adaptation in natural and artificial systems: an introductory analysis with applications to biology, control, and artificial intelligence", U Michigan Press, (1975).

[10] T. J. Pleskac, "Decision and choice: Luce's choice axiom", In J. D. Wright (Ed.), International encyclopedia of the social and behavioral sciences (Vol. 5, 2nd. ed., pp. 895-900). Oxford: Elsevier, (2015).

[11] C. M. Bishop, "Pattern Recognition and Machine Learning", Springer, (2006).

[12] H. Garmani, M. Baslam and M. Jourhmane, "Caching Games between ISP in Information Centric Network", International Journal of Control and Automation, vol. 11, no. 4, (2018), pp. 125-142.

[13] D. Ait Omar, M. Outanoute, M. Baslam, M. Fakir and B. Bouikhalne, "Joint Price and QoS Competition with Bounded Rational Customers", In: El Abbadi A., Garbinato B. (eds) Networked Systems. NETYS 2017. Lecture Notes in Computer Science, vol 10299, Springer, Cham, (2017).

[14] P. Coucheney, P. Maillé and B. Tuffin, "Impact of competition between isps on the net neutrality debate", IEEE Trans. Netw. Serv. Manag., vol. 10, pp. 425-433.

[15] J. Lorkowski and V. Kreinovich, "Bounded Rationality in Decision Making Under Uncertainty: Towards Optimal Granularity, Studies in Systems, Decision and Control", Springer International Publishing, Cham, (2018).

[16] S. Handouf, S. Arabi, E. Sabir and M. Sadik, "Telecommunication Market Share Game: Inducing Boundedly Rational Consumers via Price Misperception”, (2017). 